\title{
Inversiones en empresas con alta participación de intangibles: Estudio de casos de startups del sector TI
}

\section{Norma Pontet Ubal, Marilina Camejo, Valentina Oliver, Verónica Pérez}

\author{
Escuela de Postgrados y Ejecutivos, Facultad de Administración y Ciencias Sociales
}

Universidad ORT Uruguay

La valoración de empresas TIC en etapas tempranas resulta interesante, dado que en este estadio las mismas no cuentan con información financiera suficiente, además de que contienen una alta participación de intangibles. Esto dificulta la obtención de inversión, puesto que los métodos de valoración basados en información financiera son de difícil aplicación. Por lo tanto, el objetivo del presente trabajo es identificar cuáles son las principales variables que influyen en los aportes de capital a ser recibidos por los emprendedores en las startups del sector tecnología en Uruguay. La metodología utilizada se basa en una investigación no experimental de carácter cualitativo y de tipo exploratoria. Se identifican las principales variables tenidas en cuenta por los inversores a través de entrevistas a profundidad realizadas a algunos referentes del mercado de startups tecnológicas en Uruguay. Como resultado, se presentan las variables relevantes para los inversores a la hora de invertir en empresas TI.

Palabras clave: Tecnología de la información y la comunicación (TIC), Startup, Angel Investor, activos intangibles, valoración

\section{Investments in companies with high participation of intangibles: Case study of startups in the IT sector}

The valuation of IT companies in early stages is interesting given that at this stage they do not have sufficient financial information, in addition to containing a high participation of intangibles. This makes it difficult to obtain investment since valuation methods based on financial information are difficult to apply. Therefore, the objective of this paper is to identify which are the main variables that influence the contributions of capital to be received by entrepreneurs in startups in the technology sector in Uruguay. The methodology used is based on a non-experimental research of a qualitative and exploratory nature. The main variables taken into account by investors are identified through in-depth interviews, conducted with some market referents in order to explore the answers obtained in eight startup companies in Uruguay. As a result, relevant variables for investors when investing in IT companies are presented.

Keywords: Information and Communication Technology, Startup, Angel Investor, intangible assets, valuation

\section{Investimentos em empresas com alta participação de Intangíveis: Estudo de caso de startups no setor de TI}

A avaliação das empresas de TI em estágios iniciais é interessante, uma vez que nesta fase não possuem informações financeiras suficientes, além de conter uma alta participação de intangíveis. Isso dificulta a obtenção de investimentos, uma vez que os métodos de avaliação baseados em informaçôes financeiras são difíceis de aplicar. Portanto, o objetivo deste artigo é identificar quais são as principais variáveis que influenciam as contribuiçôes de capital a serem recebidas pelos empreendedores em startups do setor de tecnologia no Uruguai. A metodologia utilizada baseia-se em uma 
pesquisa não experimental de natureza qualitativa e exploratória. As principais variáveis consideradas pelos investidores são identificadas por meio de entrevistas em profundidade, realizadas com alguns referentes de mercado, a fim de explorar as respostas obtidas em oito startups do Uruguai. Como resultado, é apresentado as variáveis relevantes para investidores ao investir em empresas de TI.

Palavras-chave: Tecnologia da Informação e Comunicaçáo, Startup, Investidor Anjo, Ativos Intangíveis, avaliação

\section{Introducción}

Uruguay logra una temprana inserción internacional que comienza en la década de 1980, tiene un elevado ascenso en la década de 1990 y continúa hasta la actualidad con un crecimiento continuado, que lo posiciona como el primer país exportador de sistemas de información per cápita en América Latina. Las exportaciones de servicios crecen consistentemente: se han multiplicado por cuatro en los últimos años. Entre ellas, destacan los servicios no tradicionales, principalmente, servicios globales que incluyen servicios de TI, investigación y desarrollo (I\&D), servicios de negocios y financieros, entre otros. Para este tipo de servicios no tradicionales, Uruguay se ha convertido no solo en receptor de inversión extranjera directa (IED), sino que ha logrado convertirse en emisor de la misma. De acuerdo con la información relevada por la Cámara Uruguaya de Tecnologías de la Información (CUTI 2014) mediante su encuesta anual, la industria TIC uruguaya tiene una importante presencia internacional a través de filiales en el exterior: un $28 \%$ de las empresas uruguayas tienen presencia directa en el exterior, mientras que un $15 \%$ cuenta con presencia de tipo indirecta, es decir, a través de socios de negocios, distribuidores y/o representantes. Casos exitosos, en este sentido, son GeneXus, Quanam y PedidosYa.

La Tabla 1 muestra cómo las operaciones de combinaciones de negocios en TI son cada vez más frecuentes. Sin embargo, los criterios tradicionales utilizados para valoraciones de empresas no reflejan los montos que, finalmente, se terminan pagando y las diferencias suelen ser muy significativas.

\section{Tabla 1. Fusiones y adquisiciones de TIC} en Uruguay

\begin{tabular}{|c|c|c|c|c|}
\hline \multicolumn{5}{|c|}{ F\&AE INVERSION ES EN EMPRESAS TIC URUGUAYAS } \\
\hline Proceso & Año & $\begin{array}{c}\text { Empresa } \\
\text { Uy }\end{array}$ & $\begin{array}{c}\text { Empresa } \\
\text { Extranjera }\end{array}$ & $\begin{array}{l}\text { País de } \\
\text { Origen }\end{array}$ \\
\hline Adquisición & 2014 & PedidosYa & Delivery Hero & Alemania \\
\hline Integración & 2013 & PayTrue & EFTGroup & Chile \\
\hline Adquisición & 2012 & Crovat & Batanga Media & EEUU \\
\hline Adquisición & 2012 & Gproxy & Netsuite & EEUU \\
\hline Adquisición & 2012 & IPcom & $\begin{array}{l}\text { Mobileum (ex } \\
\text { Roamware) }\end{array}$ & EEUU \\
\hline $\begin{array}{l}\text { Alianza } \\
\text { estratégica y } \\
\text { fusión }\end{array}$ & 2012 & $\begin{array}{l}\text { Top } \\
\text { Systems }\end{array}$ & Stefanini & Brasil \\
\hline Adquisición & 2012 & Cubox & $\begin{array}{l}\text { NewContext- } \\
\text { Digital Garage }\end{array}$ & Japón \\
\hline Adquisición & 2012 & Southlabs & Infragistics & EEUU \\
\hline Inversión & 2011 & PedidosYa & $\begin{array}{l}\text { Atómico } \\
\text { Ventures }\end{array}$ & Inglaterra \\
\hline Inversión & 2011 & PedidosYa & $\begin{array}{l}\text { Kaszek } \\
\text { Ventures }\end{array}$ & Argentina \\
\hline Inversión & 2010 & Scanntech & $\begin{array}{l}\text { Austral } \\
\text { Capitals }\end{array}$ & Chile \\
\hline Inversión & 2010 & Scanntech & $\begin{array}{l}\text { Sequoia } \\
\text { Capital }\end{array}$ & EEUU \\
\hline Adquisición & 2008 & INI & Geodesic & India \\
\hline
\end{tabular}

Fuente: Uruguay XXI (2014). 
Las fusiones y adquisiciones (F\&A) se refieren a un aspecto de la estrategia corporativa que se ocupa de la combinación y la adquisición de otras compañías, así como de otros activos. La decisión de realizar una fusión o una adquisición es de tipo económica. Específicamente, consiste en una decisión de inversión, en que la asignación de recursos es entendida como la esperanza de obtener ingresos futuros que permitan recuperar los fondos invertidos y lograr un cierto beneficio.

\section{Ciclo de vida de las empresas y financiación}

Previo a realizar el análisis de los métodos de valuación de las empresas, debemos identificar en qué etapa del ciclo de vida se encuentran las mismas. A continuación, se presenta la Figura 1, que muestra la asociación entre el ciclo de vida de las empresas, los ingresos y la financiación para emprendimientos con mayor visibilidad en TI. Durante el estadio inicial o fase de nacimiento, la startup adopta por naturaleza una estructura pequeña y, en la mayoría de los casos, informal. Allí se desarrolla un centro de decisión altamente centralizado, a veces muy rudimentario, en una etapa de gran innovación.

Figura 1. Ciclo de vida de las empresas

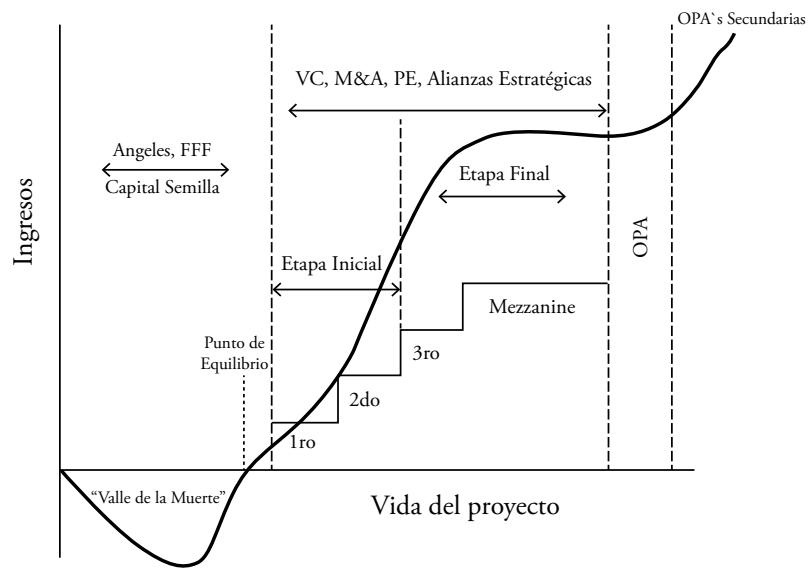

Fuente: Celis (2012).
La búsqueda de un posicionamiento o un nicho de mercado es el objetivo. Se parte de una idea de negocio que necesariamente genera pérdidas. En esta etapa, Celis (2012) menciona que los medios que tienen las empresas para financiarse son muy escasos. Los mismos se limitan a familiares, amigos o gente cercana al emprendedor. En inglés, se denomina con las siglas FFF, que significan Friends, Family and Fools (la traducción al castellano sería «Amigos, Familia y Tontos»). Solicitar un préstamo bancario es prácticamente imposible en este estadio de las empresas. Más de la mitad de las empresas se quedan en el camino, una cifra que depende del sector y del país que se considere. Pontet (2014) establece como formas de financiamiento tradicional que pueden tener los emprendedores ahorros personales y financiamiento de allegados (FFF), préstamos bancarios, tarjetas de crédito, capital proveniente de organizaciones e instituciones orientadas a promover el desarrollo de emprendedores, capital semilla e inversiones ángeles («Angel Investor» en inglés), entre otros.

Un Angel Investor es un inversor que toma sus propias decisiones de inversión, y que aporta su propio dinero y, en ocasiones, su tiempo a empresas no cotizadas promovidas por personas que le son ajenas. Aunque invierte en cualquier etapa del desarrollo, el Angel Investor desempeña un papel fundamental en la creación de empresas innovadoras al apoyar a los emprendedores en las fases iniciales del ciclo de vida de sus empresas. Buscan ganar dinero, aunque esta no sea su única motivación para invertir, ni siquiera la principal (Asociación Española de Business Angels 2016).

Durante la siguiente fase — la de crecimiento-, se incrementa la facturación sobre la base de un nicho explotado, se delegan más las decisiones, se mejoran los productos y se amplían los mercados tanto 
geográficamente como de forma horizontal a través de la incorporación de otros productos o servicios. En esta etapa del ciclo, la organización, que hasta entonces era manejada por el equipo fundador, comienza a tener que incorporar gerentes profesionales: se profesionaliza el management y se cubren puestos que antes eran cubiertos por las mismas personas. En esta etapa, se amplía la estructura, ya partiendo de una base más estable y profesional, con la aplicación de políticas internas más desarrolladas.

Según Celis (2012), en la etapa de crecimiento, existe una segunda fase de inversión en la compañía. La misma es muy probable que se realice por la vía de los denominados Angel Investors, definidos anteriormente. En esta fase, el o los inversores exigen, además de un plan de negocios y proyecciones más detalladas, una participación importante dentro de la empresa. Estas inversiones son consideradas de alto riesgo, por lo cual el inversor exigirá retornos mayores. Por esta razón, los dueños de las startups deben conocer la experiencia con la que cuenta este tipo de inversor y la industria en la que se especializa para, de esta forma, garantizar que ambas partes vayan en el mismo sentido.

Otro tipo de instituciones que suelen hacer inversiones en empresas en etapas tempranas son las empresas de Venture Capital (VC). Una diferencia entre los Angels Investors y las Venture Capital es que las segundas generalmente inyectan mayores cantidades de dinero a las compañías por medio de un fondo de inversores que, a su vez, invierte en un portafolio de diferentes compañías para diversificar su exposición al riesgo. Puede existir una etapa de reinvención de la empresa antes de alcanzar la etapa de madurez, en la que se crean nuevos productos totalmente diferentes y se exploran nuevos mercados a nivel geográfico. Se puede dar una desburocratización de la empresa a través de la descentralización de decisiones. Se da lugar a la innovación en procesos, productos, organización y marketing/ventas sobre la base de un cambio sustancial en los objetivos básicos o la esencia misma de la empresa.

En la fase de madurez, se alcanza el tamaño óptimo en el mercado. La competencia con otras empresas es máxima, debido a que otras intentarán copiar el éxito propio o viceversa. La empresa buscará hacer crecer el negocio que ya está maduro adoptando nuevas ideas externas (ya no tanto internas) de manera orgánica replicando a escala el éxito ya obtenido para multiplicar la producción, o bien a través de fusiones y/o adquisiciones de la competencia. El riesgo de burocratización es alto, lo que lleva a ineficiencias importantes, sobre todo, cuando la abundancia de cash (flujo de caja) ejercicio tras ejercicio es elevada. En este período, se da un refinamiento del producto y una mayor segmentación del mercado. En esta etapa, el margen de maniobra para crecer es menor y se cuida al accionista a través de programas de recompra de acciones, dividendos, o compensaciones salariales y premios. Ya no se es tan agresivo, sino que se adopta una estrategia, más bien, defensiva. El objetivo es alcanzar la mayor eficiencia de costos y de procesos: se agrega valor en los productos, se cuidan los márgenes, se mejoran el servicio de posventa y la imagen.

\section{Activos intangibles}

En las empresas del sector TIC, la investigación y el desarrollo, así como el potencial del emprendedor son los que le dan la esencia al negocio. Estos intangibles presentan limitaciones al momento de su reconocimiento y medición en los estados financieros, por lo que estos últimos pueden no ser una herramienta adecuada para la toma de decisiones de inversión.

Contabilidad y Negocios (14) 27, 2019 / ISSN 1992-1896 
El IASB (2009) define un activo intangible como "Aquel activo identificable no monetario sin apariencia física». Por otro lado, para el FASB (2011), un activo intangible es "Aquel activo que carece de sustancia o esencia física y tiene larga duración». Estas definiciones, sin embargo, deben ser interpretadas conjuntamente con la definición de activo. De acuerdo con el «Marco conceptual» del IASB, un activo es definido como «Un recurso controlado por la empresa como resultado de sucesos pasados y del que la entidad espera obtener en el futuro beneficios económicos» (2009).

La OCDE (2010) define que los activos intangibles son inversiones a largo plazo distintas de la compra de activos fijos y que están dirigidos a incrementar los resultados futuros de la empresa. Por su parte, para Deng y Lev (2006), los activos intangibles son fuentes no físicas de probables beneficios económicos futuros, adquiridos o desarrollados internamente con costos identificables, vida finita, valor de mercado separado de la empresa, y poseídos o controlados por la entidad.

Si bien los activos intangibles en los estados financieros son utilizados por los inversores, su uso no depende de las características del inversor, debido a que los intangibles más utilizados a la hora de invertir en una startup son el capital humano, la experiencia del emprendedor, y el empuje y la pasión del mismo. Por ser de difícil medición, estos aspectos no son aceptados por las NIIF y, por lo tanto, no se ven reflejados en los estados financieros (Smith y Cordina, 2014).

Para Cazavan-Jeny y otros (2011), entre los principales aspectos a ser considerados por un Angel Investor a la hora de invertir, se encuentra la subjetividad. En la medida que los negocios en etapa inicial son difíciles de evaluar, los modelos existentes de valuación sirven solo como indicativo. Dada la alta presencia de intangibles en las empresas TIC y dado que la contabilidad no refleja su valor adecuadamente, por las restricciones que las NIIF presentan para el reconocimiento de los mismos, los estados financieros de estas empresas pueden no ser la herramienta adecuada para la toma de decisiones.

El problema radica en que, para el caso de las empresas en estudio, estos activos son parte fundamental del negocio y son los que determinan el valor de mercado. Dadas las limitaciones que plantea la contabilidad financiera en cuanto a su reconocimiento y medición, los métodos de valuación tradicionales basados en los estados financieros presentan determinadas restricciones para la obtención de información relevante para la toma de decisiones de inversión.

\section{Metodología}

Se concibe el estudio de casos contemporáneos como una estrategia de investigación dirigida a comprender las dinámicas presentes en contextos singulares (Eisenhardt 1989). Dicha estrategia puede aplicarse para el estudio de un único caso o de varios casos combinando distintos métodos para el recojo de evidencia cualitativa y/o cuantitativa con el fin de describir, verificar o generar teoría (Eisenhardt 1989).

En la etapa inicial (etapa temprana de desarrollo de negocio), así como en las etapas subsiguientes, se recurre a la opinión de inversores y empresarios del medio relacionados con la incubación de empresas con el fin de recabar información que permita el entendimiento del sector. La investigación se desarrolla comenzando con entrevistas a profundidad a los expertos del mercado TIC en Uruguay sobre las variables que ellos toman en cuenta a la hora de invertir o incubar un nuevo proyecto. Los referentes de la industria desde los Venture Capital corresponden a Thales Lab, Endeavor, 
Centro de Emprendimientos ORT, y Agencia Nacional de Investigación e Innovación. A su vez, entre los emprendedores, se analizan diez casos, de los cuales dos fueron descartados por ser improcedentes. Para el recojo de esta información, se realizaron entrevistas semiestructuradas, con preguntas formuladas a partir de la revisión bibliográfica y de los resultados obtenidos desde los Venture Capital. Además, se llevó a cabo un intercambio abierto para obtener información de contexto y variables adicionales que no hubieran sido consideradas previamente. De la información obtenida, se formularon diez proposiciones que fueron puestas a prueba en ocho empresas que son o fueron emprendimientos. Dichas proposiciones se sustentaron en los modelos utilizados y estructurados por los diversos actores buscando la fiabilidad de procedimiento, la validez contextual y la transferibilidad en el diseño del estudio de casos.

El estudio es descriptivo, porque pretende ilustrar la situación de las startups al momento de conseguir financiamiento mediante cada uno de los casos analizados para empresas de tecnología. Asimismo, es exploratorio, puesto que pretende dar respuesta a preguntas como "¿Qué aspectos valoran los inversores al momento de invertir en startups de tecnología?» y «¿Los emprendedores buscan inversores que solo provean de financiamiento?».

\section{Objetivo y proposiciones de estudio}

El objetivo de este estudio es identificar las variables que influyen significativamente en los aportes de capital a ser recibidos por los emprendedores en las startups del sector Tecnología en Uruguay. Seguidamente, a partir de la información recabada, se ordenan las variables de acuerdo con el orden de relevancia establecida por los entrevistados y se realiza un ranking.
A continuación, se detallan las proposiciones estudiadas:

- Proposición 1: Los aportes que reciben las empresas de tecnología en Uruguay en su etapa startup provienen de los llamados FFF.

- Proposición 2: La idea de negocio es fundamental a la hora de invertir.

- Proposición 3: Las habilidades técnicas y la experiencia relacionada con la idea de negocio influyen positivamente en la obtención de financiamiento.

- Proposición 4: El negocio debe ser escalable a nivel internacional para lograr inversión para el crecimiento.

- Proposición 5: La tasa de éxitos/fracasos del emprendedor influye positivamente en los inversores.

- Proposición 6: La empatía entre el inversor y el emprendedor influye positivamente al recibir financiamiento.

- Proposición 7: En el inversor, influye positivamente que el emprendedor tenga dedicación full time al desarrollo del emprendimiento.

- Proposición 8: Para obtener financiamiento, el emprendedor debe haber validado su producto o servicio.

- Proposición 9: En la etapa de crecimiento y consolidación, los emprendedores buscan inversores que tengan conocimiento del negocio en el que se enmarca la idea.

- Proposición 10: El emprendedor debe ser flexible ante cambios que puedan surgir relacionados con el proyecto original.

Contabilidad y Negocios (14) 27, 2019 / ISSN 1992-1896 


\subsection{Casos de estudio}

ReservaTelo (Reservateloapp.com) es una aplicación para reservar hoteles de alta rotatividad en tiempo real de forma segura, anónima y gratuita.

Tabla 2. ReservaTelo

\begin{tabular}{|l|l|}
\hline Inicio de actividad & Marzo 2016 \\
\hline Fundadores & $\begin{array}{l}\text { Victoria Ortíz, Victoria Suárez y } \\
\text { Ximena Genta }\end{array}$ \\
\hline Incubados & Da Vinci Labs \\
\hline Operaciones & $\begin{array}{l}3500 \text { descargas de la App por mes (60\% } \\
\text { de los moteles operan con la App) }\end{array}$ \\
\hline Personas involucradas & 5 \\
\hline Ventas & 43000 reservas efectivas por mes \\
\hline Misión & $\begin{array}{l}\text { Apunta a promover positivamente } \\
\text { la imagen de los moteles y atraer al } \\
\text { público más joven con una comu- } \\
\text { nicación nueva, fresca y sobre todo } \\
\text { atractiva, que convierta el tema en algo } \\
\text { interesante para el usuario. }\end{array}$ \\
\hline
\end{tabular}

Fuente: Reservatelo

GPS GAY (gpsgay.com) es una plataforma uruguaya especialmente diseñada para la comunidad gay. Es la mayor comunidad online LGBT de Latinoamérica y ya se está extendiendo al resto del mundo. Cuentan con todo lo que la comunidad gay requiere en un solo lugar: mapa con geolocalización para ver usuarios cercanos, así como los lugares y eventos gay de cada ciudad, salas de chat, películas y series de temática LGBT, artículos de interés, últimas noticias, juegos y más.

Rural (rural.com.uy) es una aplicación que brinda al productor rural toda la información acerca de negocios rurales. Cuenta con resumen de noticias, información de mercados, agenda de escritorios y listado de remates, entre otros servicio.
Tabla 3. GPS GAY

\begin{tabular}{|l|l|}
\hline Inicio de actividad & Setiembre 2013 \\
\hline Fundadores & $\begin{array}{l}\text { Rosario Monteverde y Magdalena } \\
\text { Rodríguez }\end{array}$ \\
\hline Incubados & Ingenio \\
\hline Operaciones & 500000 usuarios en 2016 \\
\hline Personas involucradas & 10 \\
\hline Misión & $\begin{array}{l}\text { Apunta a ser un orientador a la hora } \\
\text { de elegir un hotel o un boliche de tipo } \\
\text { gay-friendly. Asimismo, busca promo- } \\
\text { ver el intercambio de información } \\
\text { y dar un rol activo al usuario permi- } \\
\text { tiendo que opine sobre los lugares. }\end{array}$ \\
\hline
\end{tabular}

Fuente: GPS Gay

Tabla 4. Rural

\begin{tabular}{|c|c|}
\hline Inicio de actividad & Agosto 2015 \\
\hline Fundadores & Mateo Capdevielle \\
\hline Incubados & CIE Universidad ORT \\
\hline Operaciones & $\begin{array}{l}3000 \text { visitas mensuales; } 210 \text { empresas } \\
724 \text { remates }\end{array}$ \\
\hline Personas involucradas & 3 \\
\hline Misión & $\begin{array}{l}\text { Busca brindarles una salida online a } \\
\text { todos los escritorios rurales del país } \\
\text { para posibilitar que lleven su mercado } \\
\text { a todo el territorio nacional. } \\
\text { Con la tecnología a nuestro favor, con- } \\
\text { tamos con más de } 50 \text { escritorios traba- } \\
\text { jando con nosotros. } \\
\text { Para los productores, la misión es brin- } \\
\text { darles tecnología y ponerla a trabajar } \\
\text { para ellos; de este modo, se espera } \\
\text { acercarles toda la información de la } \\
\text { manera más simple posible. }\end{array}$ \\
\hline
\end{tabular}

Fuente: Rural

Wupiti! ' (wupiti.com) es una aplicación de celulares que brinda descuentos, beneficios o regalos a los usuarios que comparten sus compras con sus amigos en una red social.

Si bien al momento de la realización del presente trabajo la empresa ya había dejado de operar en el mercado, se identifica como trabajo válido para efectos del presente estudio y se incluye en el análisis. 
Tabla 5. Wupiti

\begin{tabular}{|l|l|}
\hline Inicio de actividad & Diciembre 2013 \\
\hline Fundadores & Esteban Siniscalco y Martín Gorlero \\
\hline Incubados & CIE Universidad ORT \\
\hline Operaciones & N/A \\
\hline Personas involucradas & 2 \\
\hline Misión & $\begin{array}{l}\text { Busca lograr esa expresión de alegría } \\
\text { al conseguir algo con un descuento, } \\
\text { beneficio u obtener un regalo. }\end{array}$ \\
\hline
\end{tabular}

Fuente: Wupiti

Woow (woow.com.uy) es la tienda online más grande del país.

\section{Tabla 6. Woow}

\begin{tabular}{|l|l|}
\hline Inicio de actividad & 2006 \\
\hline Fundadores & $\begin{array}{l}\text { Leonardo Silveira, Martín Larre, Mar- } \\
\text { tín Guira }\end{array}$ \\
\hline Incubados & CIE \\
\hline Operaciones & Más de 400.000 por mes \\
\hline Personas involucradas & 160 \\
\hline Misión & $\begin{array}{l}\text { Nuestro norte es que todas las perso- } \\
\text { nas en Uruguay, cada vez que quie- } \\
\text { ran comprar algo para la casa, hacer } \\
\text { algún servicio para entretenerse, salir } \\
\text { a comer, ir al teatro, la peluquería o } \\
\text { viajar, piensen en Woow. Estamos } \\
\text { convencidos de que podemos dar un } \\
\text { servicio como el de Amazon: que, si } \\
\text { una persona quiere un cuchillo para } \\
\text { cocinar hoy de noche, pueda entrar } \\
\text { en Woow, comprarlo para esa misma } \\
\text { noche y obtener su producto. }\end{array}$ \\
\hline
\end{tabular}

Fuente: Woow

PayTrue Solutions (paytrue.com) ofrece soluciones modernas, flexibles y robustas que se adaptan rápida y fácilmente a los cambios y las demandas del mercado. Se trata de propuestas que permiten a las empresas resolver o mejorar sus medios de pago.
Tabla 7. PayTrue Solutions

\begin{tabular}{|l|l|}
\hline Inicio de actividad & 2003 \\
\hline Fundadores & $\begin{array}{l}\text { Mario Sánchez, Gonzalo Paez, Diego } \\
\text { Nario }\end{array}$ \\
\hline Incubados & $\begin{array}{l}\text { No, en 2013, hicieron una alianza de } \\
\text { integración con EFTGroup. }\end{array}$ \\
\hline Operaciones & - \\
\hline Personas involucradas & 100 \\
\hline Misión & $\begin{array}{l}\text { Busca diseñar soluciones que acompa- } \\
\text { nen los constantes cambios del mundo } \\
\text { de los medios de pago con dos obje- } \\
\text { tivos: i) promover el desarrollo perso- } \\
\text { nal, el trabajo en un ambiente laboral } \\
\text { amigable que facilite la comunicación } \\
\text { y el intercambio de conocimientos, y } \\
\text { ii) asumir con responsabilidad la tarea } \\
\text { diaria y el compromiso con el cliente } \\
\text { para alcanzar la mejora continua en la } \\
\text { gestión, así como mantener su tradi- } \\
\text { ción de satisfacción y calidad. }\end{array}$ \\
\hline
\end{tabular}

Fuente: Pay True

Sagal Solutions SRL (sagal.io) ofrece una plataforma de gestión de los canales de venta administrados por el usuario que automatiza los procesos que se realizarán para su plataforma web. Esta última está sincronizada con el sistema de gestión de la empresa.

Tabla 8. Sagal Solutions SRL

\begin{tabular}{|l|l|}
\hline Inicio de actividad & Junio2016 \\
\hline Fundadores & $\begin{array}{l}\text { Alejandro Tocar, Lorena Jinchuk, Alan } \\
\text { Burkstein, Simon Macadar, Gal Bren- } \\
\text { ner }\end{array}$ \\
\hline Incubados & Sinergia \\
\hline Operaciones & 2 clientes operan con la App \\
\hline Personas involucradas & 4 \\
\hline Misión & $\begin{array}{l}\text { Busca optimizar el tiempo de todos los } \\
\text { procesos y que la gente que se encarga } \\
\text { de realizarlos pueda realizar otras acti- } \\
\text { vidades que potencien más los canales } \\
\text { de venta online }\end{array}$ \\
\hline
\end{tabular}

Fuente: Sagal Solutions 
Ebizmarts (ebizmart.com) ofrece un software, descargable como aplicación para dispositivos con sistema operativo IOS de Apple, que conecta el stock de una o más tiendas con el del sitio web. Es un software para retail dirigido a los vendedores.

\section{Tabla 9. Ebizmarts}

\begin{tabular}{|l|l|}
\hline Inicio de actividad & 2006 \\
\hline Fundadores & Ignacia De Paula, Eli Barnett \\
\hline Incubados & No \\
\hline Cantidad de usuarios & Clientes en 18 países \\
\hline Cantidad de empleados & 18 \\
\hline Misión & $\begin{array}{l}\text { Busca lograr la atención excepcio- } \\
\text { nal al cliente y ayudar a nuestros } \\
\text { socios para aprovechar el poder de } \\
\text { Magneto. }\end{array}$ \\
\hline
\end{tabular}

Fuente: Ebizmarts
En la tabla 10, se observa que las proposiciones que se cumplen para todos los casos observados son las proposiciones $6,7,8$ y 10. La empatía entre el inversor y el emprendedor ${ }^{2}$, la dedicación full time del emprendedor en el proyecto, el haber podido validar el producto o servicio, y la flexibilidad de los emprendedores hacia los posibles cambios agregan valor a la empresa a la hora de ser evaluada para una inversión.

En relación con la proposición 4, entendemos que la misma es igual de relevante que las anteriores, aunque no se cumpla para uno de los casos. El caso inválido es el de Woow, debido a que esta empresa está focalizada en Uruguay y su objetivo es perfeccionarse en este mercado. Sin embargo, para el resto de los casos y para los expertos de mercado consultados, esta característica es una de las principales.

Tabla 10. Resultados obtenidos por cada caso analizado

\begin{tabular}{|c|c|c|c|c|c|c|c|c|}
\hline & Reservatelo & Gpsgay & Rural & Wupiti & Woow & Paytrue & Sagal & Ebizmart \\
\hline Proposición 1: FFF & Se cumple & Se cumple & Se cumple & $\begin{array}{l}\text { No se } \\
\text { cumple }\end{array}$ & No se cumple & Se cumple & $\begin{array}{l}\text { No se } \\
\text { cumple }\end{array}$ & $\begin{array}{l}\text { No se } \\
\text { cumple }\end{array}$ \\
\hline $\begin{array}{l}\text { Proposición 2: Idea de } \\
\text { negocio }\end{array}$ & $\begin{array}{l}\text { No se } \\
\text { cumple }\end{array}$ & $\begin{array}{l}\text { Se cumple } \\
\text { parcial- } \\
\text { mente }\end{array}$ & $\begin{array}{l}\text { Se cumple } \\
\text { parcial- } \\
\text { mente }\end{array}$ & $\begin{array}{l}\text { Se cumple } \\
\text { parcialmente }\end{array}$ & Se cumple & $\begin{array}{l}\text { No se } \\
\text { cumple }\end{array}$ & Se cumple & $\begin{array}{l}\text { No se } \\
\text { cumple }\end{array}$ \\
\hline $\begin{array}{l}\text { Proposición 3:Habilidades } \\
\text { técnicas y experiencia }\end{array}$ & $\begin{array}{l}\text { No se } \\
\text { cumple }\end{array}$ & Se cumple & Se cumple & Se cumple & No se cumple & Se cumple & & \\
\hline Proposición 4: Escalabilidad & Se cumple & Se cumple & Se cumple & Se cumple & No se cumple & Se cumple & Se cumple & Se cumple \\
\hline $\begin{array}{l}\text { Proposición 5: Tasa de } \\
\text { éxitos/fracasos }\end{array}$ & $\begin{array}{l}\text { No se } \\
\text { cumple }\end{array}$ & Se cumple & $\begin{array}{l}\text { No se } \\
\text { cumple }\end{array}$ & Se cumple & No se cumple & Se cumple & $\begin{array}{l}\text { No se } \\
\text { cumple }\end{array}$ & Se cumple \\
\hline Proposición 6: Empatía & Se cumple & Se cumple & Se cumple & Se cumple & Se cumple & Se cumple & No aplica & \\
\hline $\begin{array}{l}\text { Proposición 7: Dedicación } \\
\text { Juíí time }\end{array}$ & Se cumple & Se cumple & Se cumple & Se cumple & Se cumple & Se cumple & Se cumple & Se cumple \\
\hline $\begin{array}{l}\text { Proposición 8: Validación } \\
\text { del producto o servicio }\end{array}$ & Se cumple & Se cumple & Se cumple & Se cumple & Se cumple & Se cumple & Se cumple & Se cumple \\
\hline $\begin{array}{l}\text { Proposición 9: Conoci- } \\
\text { miento del inversor }\end{array}$ & Se cumple & Se cumple & Se cumple & Se cumple & No se cumple & Se cumple & No aplica & Se cumple \\
\hline Proposición 10: Flexibilidad & Se cumple & Se cumple & Se cumple & Se cumple & Se cumple & Se cumple & Se cumple & Se cumple \\
\hline
\end{tabular}

2 No aplica para el caso Sagal, debido a que la inversión obtenida fue realizada por la Agencia Nacional de Investigación e Innovación, y no ha buscado ni están en proceso de búsqueda de inversores. 
En el mismo sentido, la proposición 9 se cumple para 6 de los 8. En el caso de Sagal, no aplica, porque no ha pasado por la etapa de buscar financiamiento fuera de la ANII, mientras que, en el caso de Woow, se debe a que, al momento de recibir inversión, la empresa solo consideró la necesidad de capital. Por el contrario, la proposición 2 -relacionada con la idea de negocio- es la que menos se cumple. Se entiende que esto sucede, debido a que, si bien la idea es importante, no es lo primordial. Existen varias características que el inversor va a observar más allá de la idea, pero, sin esta, no se podría ni llegar al inversor.

En cuanto a la proposición 1, se observa que esta no se cumple en todos los casos: algunos se financian con fondos de organizaciones que se dedican a fomentar el emprendedurismo en Uruguay, como es el caso de la ANII, y aquellos que se financiaron con fondos propios sin apoyo de la ANII son empresas formadas antes de que esta comenzara a fomentar este tipo de actividades. En relación con la proposición 3, «habilidades técnicas y experiencia», si bien no se cumple en 2 de los casos, a partir de las entrevistas realizadas, obtuvimos que dicho conocimiento fue adquirido sobre la marcha del negocio actual. Finalmente, la experiencia de emprendimientos previos expuesta en la proposición 5 no se muestra como una de las características más importantes, porque, en la mitad de los casos, los emprendedores no habían tenido emprendimientos anteriores.

\section{Conclusiones}

Comenzaremos mencionando los factores que se verificaron para todos los casos, como son la empatía entre emprendedor e inversor, dedicación full time, validación del producto o servicio, y flexibilidad ante cambios que puedan surgir en relación con el proyecto original. Con respecto a la empatía, la misma refiere al relacionamiento que existe entre el emprendedor y el inversor. Si bien esta característica se cumple para todos los casos, consideramos que es importante dado que, desde el momento en que el inversor decide invertir en el emprendimiento y el emprendedor acepta dicha inversión, comienza una convivencia en la que el segundo debe sentirse respaldado y el primero, confiado de que sabrá llevar adelante el proyecto. Entendemos que el emprendedor no debería sentirse presionado por el inversor, debido a que el mismo debe estar focalizado en el negocio, y sentir que es el pilar fundamental del proyecto y no un empleado más que trabaja para el inversor. En paralelo, el inversor debe tener la seguridad de que el emprendedor actuará en defensa de sus intereses desarrollando el proyecto en concordancia con las expectativas establecidas al momento de realizar el aporte.

En cuanto a la proposición referente a la dedicación full time del emprendedor, observamos que es un factor clave a la hora de que el inversor tome la decisión de invertir. Los inversores deben poder ver que los emprendedores le dedicarán la mayor parte de su tiempo al emprendimiento: deben sentir que, para los emprendedores, su proyecto es lo más importante y que, por esto, van a hacer todo lo que esté a su alcance para llevarlo adelante. Los emprendedores reafirman esta proposición; de hecho, en todo momento, aseveran que si por lo menos un miembro del equipo no se encuentra full time abocado al proyecto las probabilidades de que sea exitoso son muy escasas.

En relación con este aspecto, consideramos que la etapa de vida en la que se encuentra el emprendedor es importante, puesto que no en todas las etapas se puede contar con tiempo suficiente como para dedicarse full time al desarrollo de un proyecto. En nuestro estudio, la mayoría de los casos corresponden a emprendedores jóvenes. El caso de Wupiti! confirma esta proposición, 
en la medida que uno de los motivos principales por los que el proyecto no siguió adelante fue por responsabilidades familiares que impidieron la dedicación full time en el emprendimiento.

La validación del producto o servicio fue otras de las proposiciones que se cumplió para todos los casos. Esta supone que, por lo menos, un cliente haya comprado un producto o servicio de los desarrollados por el emprendedor. Es decir, al menos, una persona debe haber estado dispuesta a desembolsar dinero con el fin de obtener dicho producto o servicio. Esta proposición es importante en la etapa siguiente a la incubación, debido a que en dicha etapa, por lo general, el proyecto es solo una idea a ser desarrollada. A partir de las entrevistas con los referentes del mercado, se observa que los inversores prefieren invertir en un negocio cuyo producto ya se haya vendido por lo menos una vez, en detrimento de aquellos negocios cuyos productos o servicios todavía no hayan sido comprados o usados. Desde el punto de vista del emprendedor, la validación implica que el producto o el servicio ofrecido cumple con las expectativas establecidas, y también ayuda a entender si el proyecto es viable o requiere modificaciones que puedan satisfacer de mejor manera las necesidades que pretenden cubrir.

Asociado a ello, surge la proposición 10, que refiere a la flexibilidad del emprendedor ante cambios en el proyecto original. En este caso, se entiende por flexibilidad la capacidad del emprendedor de pivotear ante determinadas circunstancias que puedan surgir y que hagan que el proyecto varíe su foco. Consideramos que esta proposición implica una característica del emprendedor que el inversor valora a la hora de aportar capital. Para empezar, los inversores buscarán que los emprendedores sean capaces de aceptar las críticas o sugerencias, y de actuar en concordancia.
Entendemos que las características antes mencionadas son las más relevantes, dadas las respuestas obtenidas de las entrevistas, que se verifican para todos los casos. Sin embargo, hay otra característica que, pese a que no se verifica para todos los casos, consideramos que es tan importante como las anteriores: la escalabilidad.

La escalabilidad refiere al fuerte potencial de crecimiento del proyecto original: que se pueda internacionalizar y que sea capaz de hacer crecer los beneficios sin reinvertir en infraestructuras al mismo nivel que aumentan los ingresos. Dado que Uruguay es un mercado pequeño, entendemos que la internacionalización es un factor fundamental si el objetivo es obtener mayor rentabilidad.

Siguiendo con el orden de relevancia, sostenemos que, cuando el emprendimiento se encuentra en una etapa temprana, es importante la proposición relacionada con el interés del emprendedor por buscar inversores con conocimiento del negocio. Por lo observado en etapas tempranas, los emprendedores buscan inversores que les brinden, además de capital, contactos, conocimiento del negocio. En etapas posteriores, en las cuales las empresas ya están instaladas y lo que buscan es la expansión, ya no es tan relevante el involucramiento del inversor con el negocio, sino el capital.

Por el lado de los inversores, basándonos en las entrevistas realizadas, observamos que estos prefieren invertir en negocios en los que tienen conocimiento, puesto que se sienten más cómodos en los rubros en los que ya tienen experiencia. Otra de las características se vincula con las habilidades técnicas y la experiencia previa del emprendedor en el rubro del emprendimiento. Esta característica es importante para los inversores, en la medida que el hecho de que los emprendedores conozcan el negocio en el cual se van a desarrollar y 
cuenten con los conocimientos técnicos para hacerlo le agrega valor al proyecto. No obstante, más relevante es la capacidad del emprendedor de absorber conocimientos a medida que el negocio se va desarrollando. Asimismo, es preciso anotar que la idea de negocio no es lo primordial a la hora de obtener financiamiento. Esta proposición, en la mayoría de los casos, se cumple parcialmente, debido a que, pese a que la idea es significativa, no es lo fundamental. Si bien es el puntapié inicial para el contacto con el inversor, este va a tomar en cuenta otras características más allá de la idea a la hora de optar por dónde invertir.

Por último, las dos características que entendemos como menos relevantes dadas las respuestas obtenidas son la tasa de éxitos o fracasos que haya tenido el emprendedor, y las fuentes de financiamiento en la etapa inicial. En relación con la primera, concluimos que no es relevante, dado que en cuatro de los casos los emprendedores no contaban con emprendimientos previos. Sin embargo, en las entrevistas con expertos, se menciona que el hecho de que el emprendedor cuente con éxitos previos es más atractivo para los inversores a la hora de optar por dónde invertir. Además, le facilita al emprendedor el acercamiento a los inversores, puesto que estos pueden conocerlo a través de rondas de inversión anteriores. En ese marco, los fracasos también constituyen un factor relevante para los inversores, en la medida que la experiencia les permite a los emprendedores conocer los problemas a los que se pueden enfrentar y no repetir los errores cometidos en el pasado.

En cuanto a la segunda característica, la forma de financiamiento de las startups en el comienzo surgió como un aspecto relevante por parte de los expertos, pues el hecho de que usen fondos FFF muestra un compromiso del emprendedor con su proyecto. No obstante, en Uruguay, existe la Agencia
Nacional de Investigación e Innovación que constituye una fuente de financiamiento muy utilizada por emprendedores en etapas iniciales. Cabe destacar que, en las entrevistas con los expertos del mercado, así como con los emprendedores, se indica que, si bien en Uruguay se ha fomentado el emprendedurismo en los últimos ańos, los inversores uruguayos se caracterizan por ser conservadores a la hora de invertir y que prefieren inversiones tradicionales, como inmuebles, campos, etc.

En resumen, para los casos seleccionados, entre las características clave que los inversores tienen en cuenta a la hora de invertir, sin establecer un orden de prelación entre las mismas, se encuentran las siguientes: empatía entre el inversor y el emprendedor, dedicación full time del emprendedor al proyecto, haber obtenido validación del producto o servicio objeto del negocio, flexibilidad del emprendedor ante cambios del proyecto original, y escalabilidad. Además, en las entrevistas con los emprendedores, se mencionó que el aporte de capital no es lo único relevante, sino que también valoran el conocimiento y la experiencia de inversor en el rubro en el que se va a desarrollar el negocio, y la empatía entre el inversor y el emprendedor. Consideramos que los inversores deberían tener en cuenta estos factores que son valorados por los emprendedores a la hora de aceptar la inversión.

\section{Referencias bibliográficas}

Asociación Española de Bussines Angels (2016). Bussines Angels. Consulta: 26 de mayo de 2017. http://www. aeban.es/sector

Cámara Uruguaya de Tecnologías de la Información (2014). Informe anual del sector TI. Montevideo. Consulta: 18 de abril de 2017. http://www.ccuruguayusa. com/wp-content/uploads/2015/05/InformeEncuestaAnualdelSectorTIResumido-Cuti.pdf

Contabilidad y Negocios (14) 27, 2019 / ISSN 1992-1896 
Cazavan-Jeny, A., Jeanjean, T. y Joos, P. (2011). Accounting choice and future performance: The case of R\&D accounting in France. Journal of Accounting and Public Policy, 30(2), 145-165. https://doi.org/10.1016/j.jaccpubpol.2010.09.016

Celis, C. (2012). Guía para el emprendedor de hoy: ¿Cómo obtener financiación?. Grandes Pymes. Consulta: 12 de junio de 2017. http://www.grandespymes.com. ar/2012/05/15/guia-para-el-emprendedor-de-hoycomo-obtener-financiacion/

Deng, Z. y Baruch, L. (2006). In-process R\&D: To capitalize or expense? Journal of Engineering and Technology Management, 23, 18-32. https://doi.org/10.1016/j. jengtecman.2006.02.003

Ebizmarts. Ebizmarts. Consulta: 12 de junio de 2017. https://ebizmart.com

Eisenhardt, Kathleen (1989). Building theories from case study research. Academy of Management Review, 14(4), 532-550. https://doi.org/10.5465/amr.1989.4308385

GPS GAY. GPS Gay. Consulta: 12 de junio de 2017. https://gpsgay.com

Griliches, Zvi (1992). The Search for R\&D Spillovers. The Scandinavian Journal of Economics, 94, 29-47. https:// doi.org/10.2307/3440244

International Accounting Standards Board (IASB) (2009). Marco conceptual para la información financiera. Londres: International Accounting Standards Board.

International Accounting Standards Board (IASB) (2016). International Financial Reporting Standards IFRS 2017. Londres: International Accounting Standards Board.

Organización de Cooperación y Desarrollo Económicos (2010). Estándares de calidad para la evaluación del desarrollo. Serie Directrices y Referencias del CAD. Madrid: Organización de Cooperación y Desarrollo Económicos.
Paytrue Solutions. Pay True. Consulta: 12 de junio de 2017. https://paytrue.com

Pontet-Ubal, Norma (2014). El rol de la contabilidad en las inversiones de alta tecnología: Estudio exploratorio en Uruguay. VII Congreso de Costos del Mercosur. Montevideo.

Reservatelo. Reservatelo. Consulta: 12 de junio de 2017. https://reservateloapp.com/

Rural. Rural. Consulta: 12 de junio de 2017. https://rural. com.uy

Sagal Solutions SRL. Sagal Solutions. Consulta: 12 de junio de 2017. https://sagal.io

Smith, Julia y Cordina, Renzo (2014). The role of accounting in high-technology investments. The British Accounting Review, 46(3), 309-322. https://doi.org/ 10.1016/j.bar.2014.03.002

Uruguay XXI (2014). La industria TIC en Uruguay [Informe]. Montevideo. Consulta: 21 de junio de 2017. http://www. uruguayxxi.gub.uy/inversiones/wpcontent/uploads/ sites/3/2014/09/la_industria_tic_en_uruguay.pdf

US Financial Accounting Standards Board (2011). Statement of financial accounting standards. Connecticut: Financial Accounting Standards Board.

Woow (2017). Woow. Consulta: 12 de junio de 2017. https://woow.com.uy

Wupiti (2017). Wupiti! Consulta: 12 de junio de 2017. https://wupiti.com

Fecha de recepción: 20 de junio de 2018 Fecha de aceptación: 28 de agosto de 2018 Correspondencia: norma.pontet@ort.edu.uy marilinacamejo@gmail.com valeoliver@icloud.com perezfontes@gmail.com 